\title{
Behavioural and demographic correlates of (rossuat undiagnosed HIV infection in a MSM sample recruited in 13 European cities
}

Ulrich Marcus ${ }^{1 *}$ (D), Christiana Nöstlinger ${ }^{2}$, Magdalena Rosińska ${ }^{3}$, Nigel Sherriff ${ }^{4}$, Lorenzo Gios ${ }^{5}$, Sonia F. Dias ${ }^{6}$, Ana F. Gama', Igor Toskin ${ }^{8}$, Ivailo Alexiev', Emilia Naseva ${ }^{10}$, Susanne Barbara Schink', Massimo Mirandola ${ }^{5}$ and the Sialon II Network

\begin{abstract}
Background: Reducing the number of people with undiagnosed HIV infection is a major goal of HIV control and prevention efforts in Europe and elsewhere. We analysed data from a large multi-city European bio-behavioural survey conducted among Men who have Sex with Men (MSM) for previously undiagnosed HIV infections, and aimed to characterise undiagnosed MSM who test less frequently than recommended.
\end{abstract}

Methods: Data on sexual behaviours and social characteristics of MSM with undiagnosed HIV infection from Sialon II, a bio-behavioural cross-sectional survey conducted in 13 European cities in 2013/2014, were compared with HIVnegative MSM. Based on reported HIV-testing patterns, we distinguished two subgroups: MSM with a negative HIV test result within 12 months prior to the study, i.e. undiagnosed incident infection, and HIV positive MSM with unknown onset of infection. Bivariate and multivariate associations of explanatory variables were analysed. Distinct multivariate multi-level random-intercept models were estimated for the entire group and both subgroups.

Results: Among 497 participants with HIV-reactive specimens, 234 (47.1\%) were classified as previously diagnosed, $106(21.3 \%)$ as incident, and $58(11.7 \%)$ as unknown onset based on self-reported status and testing history. MSM with incident HIV infection were twice as likely (odds ratio $(\mathrm{OR})=2.22,95 \%$ confidence interval $(95 \% \mathrm{Cl})$ : 1.17-4.21) to have used recreational substances during their last anal sex encounter and four times more likely $(\mathrm{OR}=3.94$, 95\%Cl: 2.14-7.27) not to discuss their HIV status with the last anal sex partner(s). MSM with unknown onset of HIV infection were 3.6 times more likely $(\mathrm{OR}=3.61,95 \% \mathrm{Cl}: 1.74-7.50)$ to report testing for a sexually transmitted infection (STI) during the last 12 months.

Conclusions: Approximately one third of the study participants who are living with HIV were unaware of their infection. Almost two-third (65\%) of those with undiagnosed HIV appeared to have acquired the infection recently, emphasizing a need for more frequent testing. Men with the identified behavioural characteristics could be considered as primary target group for HIV Pre-Exposure Prophylaxis (PrEP) to avoid HIV infection. The increased odds of those with unknown onset of HIV infection to have had an STI test in the past year strongly suggests a lost opportunity to offer HIV testing.

Keywords: HIV, Men having sex with men, Europe, Biobehavioural survey, Undiagnosed HIV infection

\footnotetext{
* Correspondence: MarcusU@rki.de

'Department of Infectious Diseases Epidemiology, Robert Koch-Institute,

Berlin, Germany

Full list of author information is available at the end of the article
}

(c) The Author(s). 2018 Open Access This article is distributed under the terms of the Creative Commons Attribution 4.0 International License (http://creativecommons.org/licenses/by/4.0/), which permits unrestricted use, distribution, and reproduction in any medium, provided you give appropriate credit to the original author(s) and the source, provide a link to the Creative Commons license, and indicate if changes were made. The Creative Commons Public Domain Dedication waiver (http://creativecommons.org/publicdomain/zero/1.0/) applies to the data made available in this article, unless otherwise stated. 


\section{Background}

In recent years a range of efforts and new initiatives have been implemented across Europe to increase $\mathrm{Hu}$ man Immunodeficiency Virus (HIV) testing among key populations and to reduce the number of undiagnosed HIV infections and late diagnoses [1]. Despite some progress in terms of increased testing uptake, a recent report from the European Centre for Disease Prevention and Control (ECDC) estimates that the proportion of undiagnosed HIV among Men having Sex with Men (MSM) in six European countries is $17 \%$ [2]. This falls short of the internationally agreed goal of diagnosing at least $90 \%$ of the people who are infected [3]. Furthermore, approximately one third of HIV diagnoses among MSM in the European Union (EU) are late (CD $4<350$ cells/ $\mu$ l at diagnosis). Reducing late HIV diagnoses would result in substantial individual (i.e. reduced morbidity and mortality) and public health benefits (reduced transmission and reduction of health care costs) $[4,5]$. In addition to adverse health outcomes, late diagnoses and late initiation of antiretroviral therapy are associated with increased risks for transmitting HIV unknowingly to sexual partners.

In countries with unrestricted access to antiretroviral treatment undiagnosed HIV infections are thought to be the main sources of new HIV infections. Early diagnosis of HIV and successful treatment are thus important for the successful management of the disease in individual patients as well as major tools supporting the implementation of the WHO strategy for anti-retroviral treatment as prevention $[3,6,7]$.

The level of undiagnosed infections is driven by HIV incidence on the one hand and by the testing rate on the other. Factors increasing HIV incidence are likely to contribute to the increased level of undiagnosed infections, even among frequent testers. Additionally, barriers to testing and low testing uptake may cause accumulation of infections, including late stage infections $[8,9]$.

Little is known about the characteristics and sexual behaviours of people with undiagnosed infections. Some information can be retrieved from HIV testing sites from people newly diagnosed with HIV [10-12], although the demographic data collected and analysed are quite limited from such clinical sites [13, 14]. People presenting for testing self-select and therefore such samples may be biased. Another way to collect such information is from longitudinal cohort studies including HIV-uninfected people at risk for HIV. However, such studies are time consuming, costly, and rarely conducted in Europe. In addition, participants may not be representative of the population at risk in real world settings. Bio-behavioural studies, such as the multi-city Sialon II study, may therefore be better suited to systematically collect information on people who are unknowingly infected with HIV $[15,16]$ with more scientific rigour and fewer biases.

In general, the risk of HIV infection among MSM is associated with sexual risk practices such as the lack of condom use, number of partners with whom condomless anal intercourse is practised, drug use associated with sex, and attending gay sex venues where risky sexual behaviours are part of the sub-culture [17]. However, such risk practices are driven by a complex set of intertwined factors, ranging from the personal level factors (e.g. age, personal skills and self-efficacy, mental health) to interpersonal factors (partner dynamics, communication and negotiation on sex practices), to community and service provision-related factors (social and sexual norms, perceived homonegativity in communities; access to testing and other medical services) and structural factors (policies and legislation). The intersection of these factors is further shaped by high HIV prevalence in subgroups of MSM [18].

Barriers to HIV testing have also been extensively described in the literature: testing for HIV is more likely for individuals who perceive themselves at risk for HIV and who anticipate personal benefits from testing, while fears of consequences of receiving an HIV diagnosis hinders HIV testing. The latter has been shown to be associated with fear of discrimination and personal rejection $[19,20]$. Research has also shown that multiple social-cognitive factors (e.g. knowledge, attitudes, perceived behavioural control) play a role in explaining testing for HIV and sexually transmitted infections (STI) among MSM [20]. In addition to patient-level factors, a review demonstrated the influence of health-systems and structural factors on uptake of HIV testing [8].

The present study used data from a large multi-city European bio-behavioural survey conducted among MSM within the framework of the European Public Health Project Sialon II. The analysis has two objectives - 1) to identify factors correlating with early undiagnosed infections among testers, which are most likely driven by HIV incidence among repeat testers; and 2) to characterise groups that are not adhering to testing recommendations in order to properly inform appropriate testing campaigns targeted towards them.

\section{Methods}

\section{Study design and procedures}

Sialon II was a multi-site bio-behavioural cross-sectional survey carried out in 13 European cities. The cities were: Brussels (Belgium), Sofia (Bulgaria), Hamburg (Germany), Verona (Italy), Vilnius (Lithuania), Warsaw (Poland), Lisbon (Portugal), Bucharest (Romania), Bratislava (Slovakia), Ljubljana (Slovenia), Barcelona (Spain), Stockholm (Sweden), and Brighton (UK). In 2013/2014, 
MSM were recruited to participate in the survey using time-location-sampling (TLS) in community-based settings in nine European cities, and using respondent-driven-sampling (RDS) in social networks of MSM in four European cities (Bucharest, Bratislava, Verona, Vilnius). In TLS cities participants were recruited during 2013, in RDS cities recruitment started in 2013 and finished in 2014. Recruitment methods, study procedures, questions asked as well as sample collection and testing have been described in detail elsewhere [21]. The study protocol was approved by ethical review committees in all participating countries and by the WHO Research Project Review Panel (WHO-RP2) and the WHO Research Ethics Review Committee (WHO-ERC) before the data collection phase.

The bio-behavioural survey data generated from the Sialon II project provided the opportunity to combine data on testing history and self-reported HIV test result, and to link them with the laboratory determined HIV status to identify men with an undiagnosed HIV infection at the time of the survey implementation. In this analysis, sexual behaviours and social characteristics of these men with undiagnosed HIV infection are assessed and compared with their uninfected peers.

\section{Measures}

Participants filled in a short questionnaire and provided either an oral fluid (in TLS cities) or blood (in RDS cities) specimen for HIV antibody testing. Based on self-reported HIV status and the HIV testing result of the collected specimen, participants were classified as HIV-uninfected (nHIV), previously diagnosed with HIV infection (pHIV), and HIV-infected but as yet undiagnosed (uHIV). As far as the time of HIV diagnosis is concerned, three different patterns can be distinguished: 1) early diagnosis, when testing is predominantly triggered by symptoms of acute HIV infection and/or awareness of transmission risk; 2) intermediate diagnosis, when testing is triggered by health concerns not immediately related to acute HIV disease or transmission risk awareness; 3) late diagnosis, often triggered by symptoms or health complaints associated with compromised immune status. These three patterns may be associated with different demographic and behavioural characteristics (see below). Since data on HIV testing intentions were not collected, we used HIV testing history to distinguish between a group with likely high testing frequency and incident HIV infection (uHIVinc negative test result reported within 12 months before the study specimen was collected) and MSM who were tested a longer time ago or never tested for HIV infection of unknown onset (uHIVunk). The uHIVinc subgroup may represent pattern 1 and partially pattern 2 testers, while the uHIVunk subgroup may represent the complementary part of pattern 2 and pattern 3 testers.
The following questionnaire items were used to determine if the case had been previously diagnosed: a question whether and if yes, when the last HIV antibody test was performed and a question on the result of the last HIV antibody test. If these questions were not answered or information was inconsistent HIV status knowledge was classified as undetermined and respondents were excluded from the analysis. In addition, participants recruited in Sofia had to be excluded from the two test recency subgroup analyses because the answers were invalid due to an incorrect translation of the respective question.

\section{Lab testing of biological samples}

In line with the TLS protocols, oral fluid (OF) specimens were collected and tested for HIV antibodies using Genscreen HIV 1/2 version 2, BIO-RAD. A total IgG antibodies ELISA test Human IgG ELISA Kit $1 \times 96$, Quantitative / Immunology Consultants Laboratory was used for OF specimen testing suitability and quality control. All HIV-reactive specimens were re-tested with Vironostika HIV Ag/Ab, bioMérieux. Specimens tested positive with the first HIV ELISA test, but negative with the second were classified as negative.

MSM who participated to the survey in cities where RDS was used as recruitment method received pre/ post-test counselling during the enrolment and follow up process. Blood samples were collected and serum extracted in line with the local standard procedures. Serum samples were tested with an HIV 4th generation ELISA/ CLIA screening test. A Western blot test was used to confirm positive cases. In case of a confirmed HIV positive result, a referral procedure was put in place in line with the local standard procedures to ensure linkage to care and proper case management.

\section{Secondary variables}

Based on published literature on factors associated with HIV acquisition risk, undiagnosed HIV infection or infrequent HIV testing and late diagnosis among MSM (as mentioned in the introduction), associations of uHIV, uHIVinc and uHIVunk status were analysed with:

- Demographic variables such as age (calculated using the self-reported year of birth), education level (secondary school or lower, high school or postsecondary or university/ higher), migration status (native: born \& living in the study country; emigrant: born in the study country \& living abroad; immigrant: born abroad \& living in the study country; visitor: born \& living abroad);

- Behavioural variables such as number of sexual partners and number of partners with whom condomless anal intercourse (AI) had been practiced 
in the previous 6 months, frequency of visiting gay sex venues in the last 3 months, type and number of drugs used during last AI (categorised as alcohol; cannabis; sexual performance enhancing substances: erectile dysfunction medication and inhaled amyl nitrite; party drugs: cocaine, ecstasy, amphetamines; chemsex drugs: GHB, ketamine, mephedrone, crystal meth);

- Type of partners for last AI (steady, non-steady, more than one), self-reported HIV serostatus disclosure to the last AI partner, sexual role during last AI (top, bottom, versatile), condom use during last AI;

- HIV and STI testing in the previous 12 months, and "outness" about sexual orientation towards relatives, friends, and co-workers.

The self-administered questionnaire filled-in by the study participants is available as Additional file 1.

\section{Statistical analysis}

We conducted analyses of bivariate and multivariate associations of explanatory variables with uHIV, and the two subgroups uHIVinc and uHIVunk, using nHIV for comparison. For the two subgroups, the comparison group was also determined by their last reported HIV test date, i.e. the comparison group for uHIVinc was tested negative within the previous 12 months, and the comparison group for uHIVunk was never tested or tested more than 12 months ago.

A multivariate multi-level logistic random-intercept model (random effect of study site) was estimated to account for the hierarchical structure of the data [22]. The multi-level analysis was conducted to identify factors associated with each subgroup separately and with the combined group. Predictors associated with the outcome variable with a probability $<0.05$ were considered significant.

Stata Version 14.2 was used (College Station, TX: StataCorp LP).

The dataset used for the analysis presented in this manuscript is available as Additional file 2. The Stata syntax of the analysis is available as Additional file 3.

\section{Results}

Study sample

A detailed description of the study sample has been published in the study report [23]. At most study sites, approximately 400 men had been recruited as requested by the study protocol, with exception of Bucharest, where only 183 participants were enrolled. There were significant age differences between study sites. The proportion of study participants tested for HIV in the last 12 months before completing the study questionnaire among those not known to have been diagnosed with HIV ranged between $35.5 \%$ in Bratislava and $66.2 \%$ in Barcelona (see Table 1).

Table 1 Sialon II study participants by study site, testing history and measured HIV status after exclusion of participants already known to have HIV and with indeterminate HIV status knowledge

\begin{tabular}{|c|c|c|c|c|c|c|c|c|}
\hline city & $\begin{array}{l}\text { HIV } \\
\text { negative } \\
\text { (n) }\end{array}$ & $\begin{array}{l}\text { percentage } \\
\text { tested for HIV } \\
\text { in the last } \\
12 \text { months (\%) }\end{array}$ & $\begin{array}{l}\text { undiagnosed } \\
\text { HIV infection } \\
\text { (n) }\end{array}$ & $\begin{array}{l}\text { percentage } \\
\text { with } \\
\text { undiagnosed } \\
\text { HIV (\%) }\end{array}$ & $\begin{array}{l}\text { undiagnosed HIV, } \\
\text { negative HIV pre-test } \\
\text { within recent } \\
12 \text { months (n) }\end{array}$ & $\begin{array}{l}\text { undiagnosed HIV } \\
\text { infection, no pre-test or } \\
\text { test longer ago than } \\
12 \text { months (n) }\end{array}$ & $\begin{array}{l}\text { percentage of } \\
\text { undiagnosed HIV } \\
\text { that may not be } \\
\text { recent (\%) }\end{array}$ & $\begin{array}{l}\text { Total } \\
\text { (N) }\end{array}$ \\
\hline Barcelona & 334 & $66.2 \%$ & 21 & $5.9 \%$ & 16 & 5 & $23.8 \%$ & 355 \\
\hline Bratislava & 376 & $35.5 \%$ & 15 & $3.8 \%$ & 6 & 9 & $60.0 \%$ & 391 \\
\hline Brighton & 331 & $56.7 \%$ & 15 & $4.3 \%$ & 10 & 5 & $33.3 \%$ & 346 \\
\hline Brussels & 327 & $63.5 \%$ & 7 & $2.1 \%$ & 5 & 2 & $28.6 \%$ & 334 \\
\hline Bucharest & 146 & $42.9 \%$ & 15 & $9.3 \%$ & 7 & 8 & $53.3 \%$ & 161 \\
\hline Hamburg & 336 & $52.1 \%$ & 15 & $4.3 \%$ & 11 & 4 & $26.7 \%$ & 351 \\
\hline Lisbon & 300 & $63.8 \%$ & 29 & $8.8 \%$ & 23 & 6 & $20.7 \%$ & 329 \\
\hline Ljubljana & 329 & $50.9 \%$ & 7 & $2.1 \%$ & 5 & 2 & $28.6 \%$ & 336 \\
\hline Sofia ${ }^{a}$ & 344 & & 12 & $3.4 \%$ & & & & 356 \\
\hline Stockholm & 334 & $49.9 \%$ & 3 & $0.9 \%$ & 2 & 1 & $33.3 \%$ & 337 \\
\hline Verona & 367 & $41.9 \%$ & 10 & $2.7 \%$ & 2 & 8 & $80.0 \%$ & 377 \\
\hline Vilnius & 314 & $38.9 \%$ & 5 & $1.6 \%$ & 2 & 3 & $60.0 \%$ & 319 \\
\hline Warsaw & 346 & $57.9 \%$ & 22 & $6.0 \%$ & 17 & 5 & $22.7 \%$ & 368 \\
\hline Total & 4184 & & 176 & $4.0 \%$ & & & & 4360 \\
\hline Total w/o Sofia & 3840 & $51.9 \%$ & 164 & & 106 & 58 & $33.0 \%$ & 4004 \\
\hline
\end{tabular}

${ }^{a}$ data on recency of last HIV testing are missing for Sofia due to incorrect translation of the question in the Bulgarian questionnaire version 
Formative research conducted in preparation of the bio-behavioural survey established that HIV testing sites, including sites providing free and anonymous HIV testing and rapid testing existed in all study cities at the time when study recruitment occurred [24]. Further qualitative assessments of gay-friendliness, accessibility and acceptability of available testing services were not conducted. HIV home tests and home collection tests were unavailable.

A valid HIV test result was available for 4716 participants. The antibody test result was non-reactive for 4219 specimens, and reactive for 497 specimens (11.8\%). From the 4219 participants with non-reactive specimens 4184 (99\%) were classified as nHIV, 35 were classified as indeterminate due to conflicting or missing self-reported data on HIV infection status. From the participants with reactive specimens 234 (47\%) were classified as pHIV, $102(20.5 \%)$ as uHIVinc, and 49 (9.9\%) as uHIVunk based on self-reported infection status and testing history. Twelve participants from Sofia with undiagnosed HIV infection could not be classified in these two subgroups. The remaining 100 (20.1\%) participants with reactive specimens had to be classified as indeterminate based on questionnaire data due to incomplete information on testing history and status knowledge (e.g. non-response to the question on previous HIV test and/or test result).

A weak positive correlation between the percentage of the participants tested for HIV by study site in the recent 12 months and the percentage of undiagnosed
HIV in the study sites was observed $(r=.275$ - see Table 1).

\section{Undiagnosed HIV infections and associations with demographics and behaviours}

The distribution of all and of undiagnosed infections by age group is shown in Fig. 1.

The percentage of undiagnosed infections from all prevalent infections is approaching 50\% in age groups younger than 35 years-old and declines to less than $30 \%$ in older age groups.

Table 1 shows the distribution of undiagnosed HIV infections by study sites. The proportion of study participants with undiagnosed HIV infection ranged from $0.9 \%$ in Stockholm to 9.3\% in Bucharest. The overall proportion of undiagnosed HIV infections among men without a recent test result was almost one-third of the undiagnosed infections, ranging from $20.7 \%$ in Lisbon to $80 \%$ in Verona. The proportions of undiagnosed HIV among infrequent testers were consistently higher than $50 \%$ in the four cities Bratislava, Bucharest, Vilnius and Verona, in which RDS was used for recruitment.

Table 2 shows the reported last test dates among study participants who did not report having HIV or a last HIV test within the 12 months before they were recruited to the Sialon study.

Table 3 shows results of bivariate analysis of associations between potential explanatory variables and the outcomes 1) undiagnosed HIV infection, acquired within the previous 12 months - uHIVinc; 2) undiagnosed HIV infection of unknown onset - uHIVunk; 3) undiagnosed

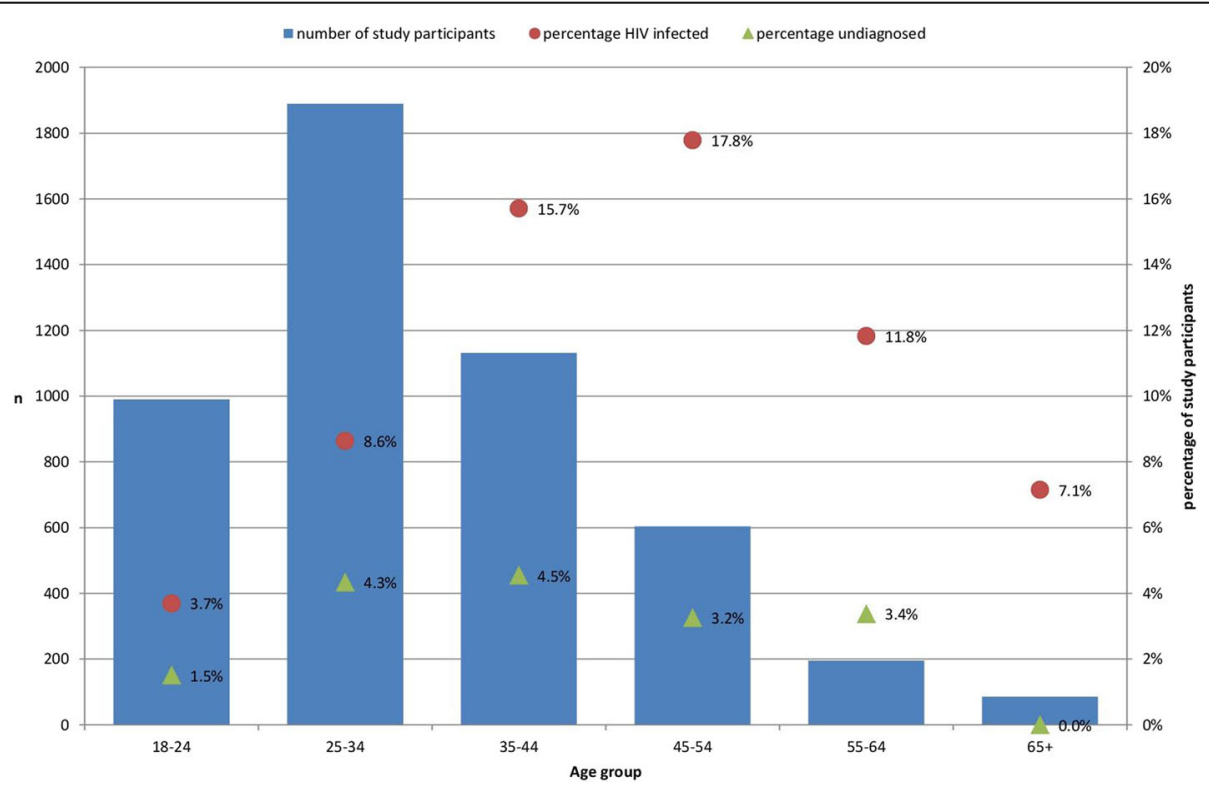

Fig. 1 Age distribution and prevalence of HIV infection and undiagnosed HIV by age group in the Sialon II participants, Sialon II biobehavioural survey, 2013-2014 
Table 2 Distribution of Sialon II study participants who were not aware of having HIV by laboratory-determined HIV status and year of last HIV test - overall and by test recency group

\begin{tabular}{|c|c|c|c|c|c|}
\hline \multicolumn{3}{|c|}{ uHIV - study sample } & \multicolumn{3}{|c|}{ uHIVunk - last HIV test not within 12 months of recruitment into the study } \\
\hline Year of last reported test & HIV negative & $\begin{array}{l}\text { HIV positive, } \\
\text { undiagnosed }\end{array}$ & Year of last reported test & HIV negative & $\begin{array}{l}\text { HIV positive, } \\
\text { undiagnosed }\end{array}$ \\
\hline 1987 & 1 & 0 & 1987 & 1 & 0 \\
\hline 1988 & 1 & 0 & 1988 & 1 & 0 \\
\hline 1989 & 2 & 0 & 1989 & 2 & 0 \\
\hline 1990 & 5 & 0 & 1990 & 5 & 0 \\
\hline 1991 & 1 & 0 & 1991 & 1 & 0 \\
\hline 1992 & 3 & 0 & 1992 & 3 & 0 \\
\hline 1993 & 2 & 0 & 1993 & 2 & 0 \\
\hline 1994 & 1 & 0 & 1994 & 1 & 0 \\
\hline 1995 & 4 & 1 & 1995 & 4 & 1 \\
\hline 1996 & 2 & 1 & 1996 & 2 & 1 \\
\hline 1997 & 5 & 1 & 1997 & 5 & 1 \\
\hline 1998 & 7 & 0 & 1998 & 7 & 0 \\
\hline 1999 & 6 & 0 & 1999 & 6 & 0 \\
\hline 2000 & 18 & 0 & 2000 & 18 & 0 \\
\hline 2001 & 10 & 0 & 2001 & 10 & 0 \\
\hline 2002 & 13 & 1 & 2002 & 13 & 1 \\
\hline 2003 & 15 & 0 & 2003 & 15 & 0 \\
\hline 2004 & 14 & 0 & 2004 & 14 & 0 \\
\hline 2005 & 39 & 2 & 2005 & 39 & 2 \\
\hline 2006 & 22 & 2 & 2006 & 22 & 2 \\
\hline 2007 & 39 & 0 & 2007 & 39 & 0 \\
\hline 2008 & 61 & 0 & 2008 & 61 & 0 \\
\hline 2009 & 80 & 4 & 2009 & 80 & 4 \\
\hline 2010 & 151 & 13 & 2010 & 151 & 13 \\
\hline 2011 & 267 & 8 & 2011 & 267 & 8 \\
\hline 2012 & 785 & 47 & 2012 & 205 & 10 \\
\hline 2013 & 1306 & 70 & 2013 & 30 & 2 \\
\hline 2014 & 32 & 0 & never tested/year missing & 864 & 13 \\
\hline year missing ${ }^{a}$ & 1292 & 26 & Total & 1868 & 58 \\
\hline \multirow[t]{7}{*}{ Total } & 4184 & 176 & & & \\
\hline & & & \multicolumn{3}{|c|}{ uHIVinc - last test within 12 months before recruitment into the study } \\
\hline & & & 2012 & 580 & 37 \\
\hline & & & 2013 & 1276 & 68 \\
\hline & & & 2014 & 32 & 0 \\
\hline & & & year missing, test within the last 12 months reported & 84 & 1 \\
\hline & & & Total & 1972 & 106 \\
\hline
\end{tabular}

includes 356 participants from Sofia whose last HIV test date could not be determined

HIV infection irrespective of date of previous HIV test (1 and 2 combined - uHIV).

Compared with HIV uninfected survey participants, men assigned to the uHIVinc group were more likely to be 25-44 years of age (compared to the reference age group 18-24), and showed higher odds for the use of drugs during last anal sex, they were less likely to have disclosed their presumed negative HIV serostatus to their last anal sex partner(s), more likely to have been versatile during their last anal sex encounter, and more 
Table 3 Associations between demographic and behaviour variables and undiagnosed HIV infection in participants of the European Sialon II biobehavioural survey

\begin{tabular}{|c|c|c|c|c|c|c|c|c|c|c|c|c|c|c|}
\hline \multirow{2}{*}{$\overline{\text { Age group }}$} & & \multicolumn{4}{|c|}{ uHIVinc } & \multicolumn{4}{|c|}{ uHIVunk } & \multicolumn{4}{|c|}{ uHIV } & \multirow[t]{2}{*}{$\mathrm{N}$} \\
\hline & & $\begin{array}{c}\text { Odds } \\
\text { Ratio } \\
\text { ref. }\end{array}$ & $\begin{array}{c}p- \\
\text { value }\end{array}$ & \multicolumn{2}{|c|}{$\begin{array}{l}\text { [95\% Conf. } \\
\text { Interval] }\end{array}$} & $\begin{array}{l}\text { Odds } \\
\text { Ratio }\end{array}$ & $\begin{array}{l}p- \\
\text { value }\end{array}$ & \multicolumn{2}{|c|}{$\begin{array}{l}\text { [95\% Conf. } \\
\text { Interval] }\end{array}$} & $\begin{array}{l}\text { Odds } \\
\text { Ratio }\end{array}$ & $\begin{array}{c}p- \\
\text { value }\end{array}$ & \multicolumn{2}{|c|}{$\begin{array}{l}\text { [95\% Conf. } \\
\text { Interval] }\end{array}$} & \\
\hline & $25-34$ & 3.34 & 0.00 & 1.58 & 7.07 & 1.69 & 0.25 & 0.70 & 4.07 & 2.65 & 0.00 & 1.56 & 4.50 & 1730 \\
\hline & $35-44$ & 3.05 & 0.01 & 1.37 & 6.80 & 2.82 & 0.02 & 1.18 & 6.73 & 2.90 & 0.00 & 1.66 & 5.06 & 970 \\
\hline & $45-54$ & 2.37 & 0.07 & 0.94 & 5.97 & 2.00 & 0.19 & 0.72 & 5.59 & 2.16 & 0.02 & 1.11 & 4.19 & 488 \\
\hline & $55+$ & 1.36 & 0.66 & 0.35 & 5.20 & 1.39 & 0.64 & 0.35 & 5.46 & 1.34 & 0.54 & 0.52 & 3.44 & 244 \\
\hline \multirow[t]{4}{*}{ Migration status } & native & ref. & & & & & & & & & & & & 3565 \\
\hline & emigrant & 2.14 & 0.20 & 0.63 & 7.20 & 6.78 & 0.02 & 1.45 & 31.84 & 3.06 & 0.01 & 1.29 & 7.27 & 54 \\
\hline & immigrant & 1.03 & 0.92 & 0.58 & 1.81 & 1.32 & 0.48 & 0.61 & 2.83 & 1.17 & 0.49 & 0.75 & 1.84 & 503 \\
\hline & $\begin{array}{l}\text { visitor: born \& live } \\
\text { abroad }\end{array}$ & 0.70 & 0.45 & 0.28 & 1.96 & 1.78 & 0.73 & 0.19 & 3.26 & 0.78 & 0.53 & 0.36 & 1.69 & 226 \\
\hline \multirow[t]{2}{*}{ Any STI test in last 12 months } & no & ref. & & & & & & & & & & & & 2249 \\
\hline & yes & 1.15 & 0.62 & 0.67 & 1.95 & 3.01 & 0.00 & 1.56 & 5.84 & 1.94 & 0.00 & 1.42 & 2.66 & 2007 \\
\hline \multirow[t]{7}{*}{ STI diagnoses } & no diagnosis & ref. & & & & & & & & & & & & 3946 \\
\hline & 1 diagnosis & 0.71 & 0.36 & 0.34 & 1.48 & 6.31 & 0.00 & 2.10 & 18.05 & 1.24 & 0.43 & 0.72 & 2.14 & 310 \\
\hline & 2 diagnoses & 1.03 & 0.97 & 0.31 & 3.35 & 4.96 & 0.14 & 0.60 & 41.01 & 1.40 & 0.52 & 0.50 & 3.88 & 74 \\
\hline & 3 diagnoses & 1.07 & 0.95 & 0.14 & 8.10 & 1.00 & - & - & - & 1.44 & 0.73 & 0.19 & 10.88 & 18 \\
\hline & 4 diagnoses & 1.00 & - & - & - & 1.00 & - & - & - & 1.00 & - & - & - & 8 \\
\hline & 5 diagnoses & 6.04 & 0.12 & 0.62 & 58.66 & 1.00 & - & - & - & 8.15 & 0.07 & 0.84 & 78.82 & 4 \\
\hline & any STI diagnosis & 0.82 & 0.52 & 0.46 & 1.49 & 5.98 & 0.00 & 2.23 & 16.06 & 1.31 & 0.26 & 0.82 & 2.09 & 414 \\
\hline \multirow[t]{6}{*}{ Number of sex partners } & no partner & ref. & & & & & & & & & & & & 269 \\
\hline & 1 partner & 0.64 & 0.38 & 0.24 & 1.72 & 4.82 & 0.13 & 0.62 & 37.34 & 1.37 & 0.46 & 0.59 & 3.17 & 819 \\
\hline & 2-3 partners & 0.78 & 0.59 & 0.31 & 1.96 & 3.73 & 0.21 & 0.47 & 29.34 & 1.34 & 0.48 & 0.59 & 3.07 & 981 \\
\hline & 4-5 partners & 0.79 & 0.63 & 0.30 & 2.08 & 4.57 & 0.16 & 0.56 & 37.45 & 1.49 & 0.36 & 0.64 & 3.51 & 626 \\
\hline & 6-10 partners & 0.67 & 0.43 & 0.25 & 1.80 & 8.35 & 0.04 & 1.08 & 64.42 & 1.62 & 0.26 & 0.70 & 3.73 & 701 \\
\hline & $>10$ partners & 0.99 & 0.89 & 0.40 & 2.47 & 10.21 & 0.03 & 1.30 & 79.90 & 2.28 & 0.05 & 1.01 & 5.14 & 748 \\
\hline \multirow{6}{*}{$\begin{array}{l}\text { Number of partners with } \\
\text { condomless anal intercourse }\end{array}$} & no partner & ref. & & & & & & & & & & & & 1499 \\
\hline & 1 partner & 0.57 & 0.06 & 0.32 & 1.02 & 1.30 & 0.54 & 0.57 & 2.96 & 0.85 & 0.49 & 0.55 & 1.33 & 1108 \\
\hline & 2-3 partners & 1.13 & 0.65 & 0.67 & 1.90 & 2.11 & 0.07 & 0.95 & 4.68 & 1.36 & 0.16 & 0.89 & 2.07 & 839 \\
\hline & 4-5 partners & 0.67 & 0.46 & 0.24 & 1.92 & 5.03 & 0.00 & 1.92 & 13.16 & 1.50 & 0.22 & 0.79 & 2.85 & 235 \\
\hline & 6-10 partners & 1.17 & 0.75 & 0.45 & 3.05 & 2.94 & 0.10 & 0.80 & 10.67 & 1.79 & 0.09 & 0.92 & 3.50 & 182 \\
\hline & $>10$ partners & 2.68 & 0.02 & 1.14 & 6.30 & 12.84 & 0.00 & 3.75 & 43.93 & 3.55 & 0.00 & 1.83 & 6.88 & 106 \\
\hline \multirow{4}{*}{$\begin{array}{l}\text { Type of partners in last } \\
6 \text { months }\end{array}$} & steady partner(s) & ref. & & & & & & & & & & & & 653 \\
\hline & $\begin{array}{l}\text { non-steady } \\
\text { partner(s) }\end{array}$ & 1.18 & 0.65 & 0.58 & 2.38 & 1.08 & 0.86 & 0.49 & 2.35 & 1.12 & 0.66 & 0.68 & 1.85 & 1297 \\
\hline & $\mathrm{s}+\mathrm{ns}$ partner(s) & 1.31 & 0.42 & 0.68 & 2.55 & 1.00 & 1.00 & 0.49 & 2.04 & 1.23 & 0.39 & 0.77 & 1.97 & 2001 \\
\hline & no partner & 1.67 & 0.35 & 0.56 & 4.97 & 0.22 & 0.15 & 0.03 & 1.71 & 0.78 & 0.60 & 0.31 & 1.95 & 216 \\
\hline \multirow[t]{3}{*}{ Anal sex in last 6 months } & no anal sex & ref. & & & & & & & & & & & & 857 \\
\hline & $\begin{array}{l}\text { anal intercourse } \\
\text { (with condom) }\end{array}$ & 0.87 & 0.65 & 0.47 & 1.59 & 1.21 & 0.67 & 0.50 & 2.95 & 1.12 & 0.65 & 0.69 & 1.84 & 1005 \\
\hline & $\begin{array}{l}\text { condomless anal } \\
\text { intercourse }\end{array}$ & 0.80 & 0.43 & 0.47 & 1.38 & 1.95 & 0.05 & 0.99 & 3.85 & 1.30 & 0.21 & 0.86 & 1.98 & 2498 \\
\hline Type of partner at last anal sex & steady partner & ref. & & & & & & & & & & & & 1880 \\
\hline & non-steady partner & 1.01 & 0.96 & 0.65 & 1.67 & 1.04 & 0.07 & 0.95 & 3.17 & 1.30 & 0.13 & 0.93 & 1.82 & 1760 \\
\hline
\end{tabular}


Table 3 Associations between demographic and behaviour variables and undiagnosed HIV infection in participants of the European Sialon II biobehavioural survey (Continued)

\begin{tabular}{|c|c|c|c|c|c|c|c|c|c|c|c|c|c|c|}
\hline & \multirow[b]{3}{*}{ more than one partner } & \multicolumn{4}{|c|}{ uHIVinc } & \multicolumn{4}{|c|}{ uHIVunk } & \multicolumn{4}{|c|}{ uHIV } & \multirow{3}{*}{$\begin{array}{l}{ }^{N} \\
207\end{array}$} \\
\hline & & \multirow{2}{*}{$\begin{array}{l}\text { Odds } \\
\text { Ratio }\end{array}$} & \multirow{2}{*}{$\begin{array}{c}\frac{p-}{} \\
\text { value }\end{array}$} & \multicolumn{2}{|c|}{$\begin{array}{l}\text { [95\% Conf. } \\
\text { Interval] }\end{array}$} & \multirow{2}{*}{$\begin{array}{l}\begin{array}{l}\text { Odds } \\
\text { Ratio }\end{array} \\
2.33\end{array}$} & \multirow{2}{*}{$\begin{array}{l}p- \\
\text { value }\end{array}$} & \multicolumn{2}{|c|}{$\begin{array}{l}\text { [95\% Conf. } \\
\text { Interval] }\end{array}$} & \multirow{2}{*}{$\begin{array}{l}\begin{array}{l}\text { Odds } \\
\text { Ratio }\end{array} \\
\mathbf{2 . 2 2}\end{array}$} & \multirow{2}{*}{$\begin{array}{c}\begin{array}{c}p- \\
\text { value }\end{array} \\
\mathbf{0 . 0 1}\end{array}$} & \multicolumn{2}{|c|}{$\begin{array}{l}\text { [95\% Conf. } \\
\text { Interval] }\end{array}$} & \\
\hline & & & & 1.03 & 4.11 & & & 0.77 & 7.04 & & & 1.24 & 3.97 & \\
\hline \multirow[t]{3}{*}{ Sexual role with last Al partner } & top & ref. & & & & & & & & & & & & 1324 \\
\hline & bottom & 1.11 & 0.71 & 0.64 & 1.94 & 1.07 & 0.93 & 0.49 & 1.94 & 0.98 & 0.93 & 0.65 & 1.48 & 1265 \\
\hline & versatile & 2.08 & 0.00 & 1.27 & 3.42 & 1.00 & 1.00 & 0.48 & 2.09 & 1.61 & 0.02 & 1.09 & 2.37 & 1035 \\
\hline \multirow{2}{*}{$\begin{array}{l}\text { Serostatus disclosure to last Al } \\
\text { partner }\end{array}$} & no disclosure & ref. & & & & & & & & & & & & 2400 \\
\hline & disclosure & 0.27 & 0.00 & 0.15 & 0.48 & 1.18 & 0.82 & 0.56 & 2.06 & 0.50 & 0.00 & 0.33 & 0.75 & 1262 \\
\hline \multirow{10}{*}{$\begin{array}{l}\text { Number of drugs consumed at } \\
\text { last anal sex }\end{array}$} & 0 & ref. & & & & & & & & & & & & 1959 \\
\hline & 1 & 2.31 & 0.00 & 1.37 & 3.91 & 0.91 & 0.75 & 0.50 & 1.66 & 1.50 & 0.03 & 1.04 & 2.16 & 1394 \\
\hline & 2 & 2.98 & 0.00 & 1.58 & 5.61 & 0.99 & 0.99 & 0.41 & 2.42 & 1.74 & 0.02 & 1.08 & 2.79 & 516 \\
\hline & 3 & 4.30 & 0.00 & 2.03 & 9.09 & 1.00 & - & - & - & 2.31 & 0.01 & 1.19 & 4.49 & 167 \\
\hline & 4 & 5.59 & 0.00 & 1.79 & 11.80 & 2.04 & 0.50 & 0.26 & 15.97 & 3.33 & 0.00 & 1.46 & 7.55 & 76 \\
\hline & 5 & 4.69 & 0.02 & 1.32 & 16.70 & 1.00 & - & - & - & 2.66 & 0.11 & 0.80 & 8.87 & 40 \\
\hline & 6 & 9.38 & 0.01 & 1.89 & 54.64 & 7.66 & 0.07 & 0.83 & 70.60 & 8.19 & 0.00 & 2.25 & 29.82 & 15 \\
\hline & 7 & 4.17 & 0.18 & 0.51 & 34.29 & 1.00 & - & - & - & 2.98 & 0.30 & 0.38 & 23.46 & 12 \\
\hline & 8 & 1.00 & - & - & - & 1.00 & - & - & - & 1.00 & - & - & - & 6 \\
\hline & 9 & 18.76 & 0.02 & 1.64 & 214.37 & 1.00 & - & - & - & 10.93 & 0.04 & 1.12 & 106.62 & 4 \\
\hline \multirow[t]{10}{*}{ Type of drugs } & no party drug & ref. & & & & & & & & & & & & 3875 \\
\hline & party & 2.90 & 0.00 & 1.77 & 4.77 & 0.36 & 0.32 & 0.05 & 2.66 & 2.04 & 0.00 & 1.29 & 3.22 & 307 \\
\hline & no chemsex drug & ref. & & & & & & & & & & & & 4063 \\
\hline & chemsex & 2.14 & 0.04 & 1.04 & 4.39 & 1.89 & 0.54 & 0.25 & 14.45 & 2.42 & 0.01 & 1.24 & 4.71 & 111 \\
\hline & $\begin{array}{l}\text { no sexual performance } \\
\text { substance }\end{array}$ & ref. & & & & & & & & & & & & 3326 \\
\hline & $\begin{array}{l}\text { sexual performance } \\
\text { substance }\end{array}$ & 2.41 & 0.00 & 1.61 & 3.61 & 1.79 & 0.07 & 0.95 & 3.37 & 2.15 & 0.00 & 1.55 & 2.98 & 853 \\
\hline & no cannabis & ref. & & & & & & & & & & & & 3869 \\
\hline & cannabis & 2.20 & 0.01 & 1.27 & 3.79 & 0.89 & 0.85 & 0.27 & 2.91 & 1.75 & 0.02 & 1.08 & 2.83 & 304 \\
\hline & no alcohol & ref. & & & & & & & & & & & & 2.324 \\
\hline & alcohol & 1.90 & 0.00 & 1.26 & 2.84 & 0.70 & 0.23 & 0.40 & 1.25 & 1.33 & 0.07 & 0.98 & 1.80 & 1859 \\
\hline \multirow[t]{2}{*}{ Satisfaction with sex life } & unsatisfied & ref. & & & & & & & & & & & & 950 \\
\hline & satisfied & 1.70 & 0.08 & 0.94 & 3.08 & 1.16 & 0.67 & 0.59 & 2.27 & 1.48 & 0.06 & 0.98 & 2.22 & 3.124 \\
\hline
\end{tabular}

likely to have had more than 10 partners in the last 6 months with whom they had condomless anal intercourse.

Men assigned to the uHIVunk group were more likely to be older (age groups 35-44) than HIV-uninfected men who had not been tested for HIV in the last 12 months, to report any condomless anal intercourse in the last 6 months, and to have higher numbers of partners in the last 6 months with whom they had condomless anal sex, they were more likely to have been tested for and diagnosed with an STI in the last 12 months, and more likely to be an emigrant on home visit to his country of origin, but they were mostly inconspicuous in terms of substance use and most other potential explanatory variables.

In multivariate analysis assignment to the uHIVinc group remained significantly associated with age 25-34, and versatility, lack of serostatus disclosure, and use of party and sexual performance enhancing drugs during the last anal sex event (see Table 4). The only factors remaining associated with uHIVunk in multivariate analysis were age 3554 , higher number of partners with whom condomless anal sex had been practiced in the last 6 months, and more frequent STI testing in the last 12 months.

Education, migration status, outness, frequency of visiting gay sex venues in the last 6 months, partnership 
Table 4 Multivariate multilevel models to estimate associations between undiagnosed ${ }^{a}$ HIV infection and demographic and behavioural parameters among participants of the Sialon II study

\begin{tabular}{|c|c|c|c|c|c|}
\hline & & Odds Ratio & $p$-value & \multicolumn{2}{|c|}{ [95\% Conf. Interval] } \\
\hline \multicolumn{6}{|l|}{ uHIVinc $(n=1713)$} \\
\hline \multirow[t]{5}{*}{ Age group } & $18-24$ & ref. & & & \\
\hline & $25-34$ & 2.27 & 0.04 & 1.03 & 4.99 \\
\hline & $35-44$ & 1.62 & 0.29 & 0.67 & 3.90 \\
\hline & $45-54$ & 1.37 & 0.56 & 0.48 & 3.96 \\
\hline & $55+$ & 1.08 & 0.91 & 0.26 & 4.46 \\
\hline \multirow[t]{3}{*}{ Sexual role with last Al partner } & top & ref. & & & \\
\hline & bottom & 1.12 & 0.73 & 0.60 & 2.07 \\
\hline & versatile & 2.05 & 0.01 & 1.18 & 3.55 \\
\hline \multirow[t]{4}{*}{ Type of drugs during last anal sex } & no use of ecstasy, cocaine, amphetamine & ref. & & & \\
\hline & ecstasy, cocaine, amphetamine & 2.22 & 0.02 & 1.17 & 4.21 \\
\hline & $\begin{array}{l}\text { no use of sexual performance substances } \\
\text { (poppers, erectile dysfunction medication) }\end{array}$ & ref. & & & \\
\hline & $\begin{array}{l}\text { sexual performance substances (poppers, } \\
\text { erectile dysfunction medication) }\end{array}$ & 1.96 & 0.01 & 1.17 & 3.28 \\
\hline \multirow[t]{2}{*}{ Serostatus disclosure to last Al partner } & disclosure & ref. & & & \\
\hline & no disclosure & 3.94 & 0.00 & 2.14 & 7.27 \\
\hline _cons & & 0.01 & 0.00 & 0.00 & 0.01 \\
\hline city & & 0.40 & - & 0.11 & 1.45 \\
\hline \multicolumn{6}{|l|}{ uHIVunk $(n=1639)$} \\
\hline \multirow[t]{5}{*}{ Age group } & $18-24$ & ref. & & & \\
\hline & $25-34$ & 2.00 & 0.16 & 0.76 & 5.27 \\
\hline & $35-44$ & 3.73 & 0.01 & 1.41 & 9.84 \\
\hline & $45-54$ & 3.31 & 0.04 & 1.08 & 10.12 \\
\hline & $55+$ & 0.90 & 0.93 & 0.10 & 7.84 \\
\hline \multirow[t]{6}{*}{ Number of partners with condomless anal intercourse } & no partner & ref. & & & \\
\hline & 1 partner & 1.46 & 0.38 & 0.63 & 3.36 \\
\hline & 2-3 partners & 2.66 & 0.02 & 1.17 & 6.04 \\
\hline & 4-5 partners & 6.08 & 0.00 & 2.26 & 16.40 \\
\hline & 6-10 partners & 2.01 & 0.38 & 0.43 & 9.44 \\
\hline & $>10$ partners & 12.83 & 0.00 & 3.60 & 45.65 \\
\hline \multirow[t]{2}{*}{ STI testing in last 12 months } & no testing & ref. & & & \\
\hline & any STI testing & 3.61 & 0.00 & 1.74 & 7.50 \\
\hline _cons & & 0.02 & 0.00 & 0.01 & 0.07 \\
\hline city & & 0.00 & - & - & - \\
\hline
\end{tabular}


Table 4 Multivariate multilevel models to estimate associations between undiagnosed ${ }^{\mathrm{a}}$ HIV infection and demographic and behavioural parameters among participants of the Sialon II study (Continued)

\begin{tabular}{|c|c|c|c|c|c|}
\hline \multirow{2}{*}{$\overline{\mathrm{uHIV}}(n=3745)$} & & \multirow[t]{2}{*}{ Odds Ratio } & \multirow[t]{2}{*}{$p$-value } & \multicolumn{2}{|c|}{ [95\% Conf. Interval] } \\
\hline & & & & & \\
\hline \multirow[t]{5}{*}{ Age group } & $18-24$ & ref. & & & \\
\hline & $25-34$ & 2.36 & 0.00 & 1.33 & 4.19 \\
\hline & $35-44$ & 2.22 & 0.01 & 1.19 & 4.13 \\
\hline & $45-54$ & 1.90 & 0.09 & 0.91 & 3.94 \\
\hline & $55+$ & 0.85 & 0.78 & 0.27 & 2.66 \\
\hline \multirow[t]{2}{*}{ HIV test in last 12 months and knowing the result } & not tested & ref. & & & \\
\hline & tested and knowing the result & 1.51 & 0.03 & 1.04 & 2.19 \\
\hline \multirow[t]{6}{*}{ Number of partners with condomless anal intercourse } & no partner & ref. & & & \\
\hline & 1 partner & 0.85 & 0.50 & 0.54 & 1.35 \\
\hline & 2-3 partners & 1.28 & 0.30 & 0.81 & 2.03 \\
\hline & 4-5 partners & 1.35 & 0.39 & 0.68 & 2.69 \\
\hline & 6-10 partners & 1.56 & 0.25 & 0.74 & 3.29 \\
\hline & $>10$ partners & 2.80 & 0.01 & 1.34 & 5.85 \\
\hline \multirow[t]{2}{*}{ Type of drugs during last anal sex } & $\begin{array}{l}\text { no use of sexual performance substances } \\
\text { (poppers, erectile dysfunction medication) }\end{array}$ & ref. & & & \\
\hline & $\begin{array}{l}\text { sexual performance substances (poppers, } \\
\text { erectile dysfunction medication) }\end{array}$ & 1.91 & 0.00 & 1.32 & 2.76 \\
\hline _cons & & 0.01 & 0.00 & 0.01 & 0.02 \\
\hline city & & 0.32 & - & 0.10 & 1.01 \\
\hline
\end{tabular}

athe three models estimate associations in three groups:

uHIVinc - undiagnosed HIV in a group of men reporting a last negative HIV test result within the previous 12 months

uHIVunk - undiagnosed HIV in a group of men who never tested for HIV or whose last negative HIV test result is older than 12 months

uHIV - undiagnosed HIV in the combined group of men irrespective of the time of the last negative HIV test

status, type of partner for the last anal intercourse, condom use during last anal intercourse, and sexual role during last anal intercourse were not significantly different between men with and without undiagnosed HIV infection.

\section{Discussion}

Approximately one third of the study participants who were living with HIV and for whom their HIV status knowledge could be assessed were unaware of being infected. This is much higher than proportions reported from some modelling studies or estimates reported to ECDC for Dublin Declaration monitoring [2, 25, 26]. This apparent contradiction is likely explained by an age related effect in our sample: as we can show in our analyses, the proportion of undiagnosed HIV is highly age-dependent. A large proportion of MSM living with HIV in the Western European countries, where the HIV epidemic amongst MSM started already in the 1980s, is already older than 40 years. These higher age groups are underrepresented among the visitors of gay venues that often cater to younger MSM clients. When the different age composition of the Sialon sample and the MSM population in modelling studies are considered, the results in terms of the proportions of undiagnosed infections are essentially comparable [own unpublished comparisons between modelling results of the German undiagnosed fraction and Sialon results for Hamburg]. Contrastingly, in Eastern European countries, where the HIV epidemic among MSM is more recent and the fraction of older infections in aging survivors is much smaller, the Sialon results are comparable with modelling studies [27]. Another aspect that needs to be considered when comparing Sialon II results with national modelling studies is that Sialon II was conducted in large cities while modelling studies include whole countries. Regardless, our findings underline that in many settings where MSM congregate and seek sexual partners, a considerable proportion of those who are living with HIV are unaware of their HIV status.

Our analysis further shows that men with an undiagnosed HIV infection are a heterogeneous group of people. In our European multi-city sample, approximately two-third of those with undiagnosed HIV infection reported to have received a negative HIV test result in the previous 12 months, indicating the relatively recent acquisition of the infection and substantial incidence in this group. Moreover, this subgroup of men 
appears to test more frequently and be aware of risks. Taking this into account, the probability is high that many of them would have been tested again and diagnosed in the near future. It might also be that some of them tested in the HIV window period and received a false negative test result. To improve early HIV diagnosis in this group, men with these characteristics presenting for HIV testing should be offered laboratory testing with 4th generation HIV antigen/antibody tests to increase the probability to detect recent infections. If sufficient resources are available, even targeted PCR testing could be considered if this subgroup can be identified among the clients of the testing facilities, e.g. based on a combined symptoms and behaviours score [28, 29].

The men with undiagnosed infection following a negative test within the past 12 months had high odds of having used recreational drugs during their last anal sex encounter and high odds of not discussing their HIV status with the last anal sex partner(s) [30]. Because viral load and transmissibility of HIV are very high during the phase of acute HIV infection [31-33], many of their recent sexual partners may have been at high risk for acquiring HIV infection if they engaged in condomless anal intercourse relying on an assumed negative HIV status. In the literature, the associations between repeat testing and risk behaviours are complex. Receiving a negative result may trigger different reactions from reassurance in safe practices to feeling lucky or invulnerable, or reinforce risky behaviour that is associated with a subsequent higher frequency of unprotected sex [34].

These findings clearly point to the need of recommending more frequent testing in selected groups of MSM, especially to those using recreational drugs. More importantly, the testers could be considered as primary target group for HIV pre-exposure prophylaxis (PrEP) to avoid HIV infection in the first place, as also suggested by other authors [35].

Approximately one-third of the men with undiagnosed HIV in the Sialon II sample infrequently test for HIV, although they tend to have multiple condomless anal sex encounters. Higher proportions were observed particularly in the four RDS cities, which may suggest that more hidden subgroups within the MSM populations were reached (see also Limitations). This, from a public health perspective, is an advantage of this sampling methodology compared to TLS method and probably to National HIV surveillance systems as well. While the study was not designed to answer the research question on identifying characteristics and behaviours of undiagnosed HIV-infected participants, only number of partners with whom condomless anal intercourse was practiced and more frequent STI testing was associated with the outcome variable (undiagnosed HIV infection) in this group. While age was significantly and independently associated with being undiagnosed in this group, more research will be necessary to characterize MSM living with undiagnosed HIV infection who do not test frequently for this infection in order to develop evidence-based interventions to increase test uptake. However, in the bivariate analysis we also found high odds for having been diagnosed with a STI during the last 12 months in this group. This strongly suggests that contrary to guidelines and recommendations HIV testing had not been offered or not been conducted in the context of these STI diagnoses. We are unable to determine whether this missed opportunity for an earlier diagnosis of HIV is related to a lack of discussion and disclosure of sexual orientation with the STI test and treatment provider or to a lack of compliance with testing guidelines by the STI treatment providers.

Partnership status and type of partner for last anal intercourse were not significantly associated with undiagnosed HIV, suggesting that condomless sex within steady partnerships may not always be as safe as people tend to assume, particularly if HIV status has not been checked mutually and/or if condomless anal sex is practiced concurrently with non-steady partners.

\section{Limitations}

For correct interpretation of our findings it must be considered that we report on associations with undiagnosed HIV infections in a very specific group. Factors associated with undiagnosed HIV may partly be different from factors associated with transmission risk, because a part of those who acquire HIV will be diagnosed and detected early. For MSM who infrequently test for HIV it may be difficult to detect behavioural correlates for their infection risk because we asked for behaviours in the previous 6 months. The moment when these men acquired HIV may be longer ago and their behaviour may have changed. MSM who have never been tested for HIV may be underrepresented in our sample. Never tested MSM are often less integrated into gay communities and rarely visit gay venues; this explains why they would have a lower chance to be recruited in our study, at least when considering the cities where a TLS approach has been adopted to enrol study participants [36]. This means that our uHIVunk group may mainly represent pattern 2 testing (triggered by health concerns not immediately related to acute HIV disease or transmission risk awareness) and less pattern 3 testing. A further limitation is that HIV status knowledge was based on self-reports and some participants may have felt uncomfortable reporting their HIV status in the questionnaire. Underreporting of a positive HIV status would have weakened any association we found between being undiagnosed and other factors.

\section{Conclusions}

Our study findings reinforce the recommendations for healthcare provider-initiated HIV testing when certain 
indicator diseases such as STIs are diagnosed. The findings may also inform community-based low-threshold HIV testing strategies such as home-collection sampling and test promotion campaigns to reduce the proportion of the hidden HIV epidemic. Such strategies should include certain elements of information (e.g. on the sensitivity of different tests during acute HIV infection), focus on interpersonal skills and community norms (e.g. communication with sexual partners about serostatus) and highlight additional risks associated with recreational drug use, while recognising the diversity of MSM with undiagnosed HIV across Europe. In addition, novel strategies such as home-testing should be discussed in the light of safeguarding linkage to care [37]. Since data were collected in different European cities, the findings allow for a high degree of tailoring local prevention campaigns, i.e. developing targeted HIV and STI testing campaigns considering the local contexts in both community-based HIV testing and counselling and advice offered at such HIV testing sites [38, 39].

More importantly, tailored strategies based on the established HIV testing patterns should be embedded within an overall combined prevention approach [40], which should include the addition of PrEP to the available effective prevention tools [40-42] for instance for those MSM reporting condomless anal sex with multiple partners in the last 6 months.

\section{Additional files}

Additional file 1: English language version of the self-administered questionnaire filled-in by the study participants. (PDF $235 \mathrm{~kb}$ )

Additional file 2: Sialon II dataset used for the analysis presented in this manuscript. (CSV $11362 \mathrm{~kb}$ )

Additional file 3: Stata do-file of the analysis. (TXT $10 \mathrm{~kb}$ )

\begin{abstract}
Abbreviations
Al: Anal intercourse; CD4: T-helper lymphocytes; CLIA: ChemoluminescenceImmunoassay; ECDC: European Centre for Disease Prevention and Control; ELISA: Enzyme Linked Immuno Sorbent Assay; EU: European Union; GHB: Yhydroxybutric acid; HIV Ag/Ab: HIV Antigen/Antibody; HIV: Human Immunodeficiency Virus; IgG: Immunoglobulin G; MSM: Men having sex with men; nHIV: HIV-uninfected; OF: Oral fluid; pHIV: Previously diagnosed with HIV infection; PrEP: Pre-exposure prophylaxis; RDS: Respondent-drivensampling; STI: Sexually transmitted infection; TLS: Time-location-sampling; uHIV: HIV-infected but as yet undiagnosed; uHIVinc: Incident HIV infection negative test result reported within 12 months before the study specimen was collected; uHIVunk: HIV infection of unknown onset; UK: United Kingdom; WHO: World Health Organization; WHO-ERC: WHO Ethics Review Committee; WHO-RP2: WHO Research Project Review Panel
\end{abstract}

\section{Acknowledgements}

The authors would like to acknowledge the contributions of the Sialon II network which made this study possible, and of all study participants who dedicated their time to fill in the questionnaire and gave a biological specimen for laboratory testing.

The Sialon II Network (extended list).

Massimo Mirandola, Lorenzo Gios, Stefano Benvenuti, Ruth Joanna Davis, Silvana Menichelli, Michele Breveglieri, (Coordinamento Regionale per il Management e la Progettazione Europea, Azienda Ospedaliera Universitaria
Integrata, Verona, Italy); Wim Vanden Berghe, Peter de Groot, Christiana Nöstlinger, Veronica van Wijk, Katrien Fransen, Tine Vermoesen, Michiel Vanackere (Institute of Tropical Medicine, ITG, Antwerp, Belgium); Fourat Benchikha, Sandra Van den Eynde, Boris Cruyssaert, Mark Sergeant, Karel Blondeel, Pieter Damen (Sensoa, Antwerp, Belgium); François Massoz, Erwin Carlier (Rainbowhouse Brussels, Belgium); Michael François, Stephen Karon (Ex Aequo, Belgium); Safia Soltani, Thierry Martin (Belgium); Alan De Bruyne (The Belgian Pride, Belgium); Francoise Bocken (Alias, Belgium); Myriam Dieleman (Observatoire du sida et des sexualités, Belgium); Ivailo Alexiev, Reneta Dimitrova, Anna Gancheva, Dobromira Bogeva, Maria Nikolova, Mariya Muhtarova, Todor Kantarjiev, (National Center of Infectious and Parasitic Diseases, Sofia, Bulgaria); Viara Georgieva (National Center of Infectious and Parasitic Diseases, Sofia, Bulgaria; Ministry of Health, Sofia, Bulgaria); Emilia Naseva, Petar Tsintsarski, Hristo Taskov, Tonka Varleva (Program "Prevention and Control of HIV/AIDS", Ministry of Health, Sofia, Bulgaria); Elena Birindjieva, Aneliya Angelova, Manol Antonov (Association "Health without borders", Bulgaria); Ulrich Marcus, Susanne Barbara Schink, Sandra Dudareva-Vizule, Matthias an der Heiden, Sami Marzougui, Viviane Bremer, Andrea Kühne, Kerstin Schönerstedt-Zastrau, Ruth Zimmermann (Robert Koch Institute, Berlin, Germany); Andreas Wille (Institut für Hygiene und Umwelt, Hamburg, Germany); Kai Eckstein, Norman Buch, Philipp Moskophidis, Marc Grenz, Danilo Schmogro, (Hein \& Fiete, Hamburg, Germany); Giuseppe Cornaglia, Antonella Zorzi, Elisabetta Tonolli, Giuliana Lo Cascio, Teresa Todeschini, Manuela Recchia, Lorella Pattini, Maria Rocca, Alessandra Bighignoli, Anita Galardi, Loredana Martini, Francesco Cobello, Chiara Bovo (Azienda Ospedaliera Universitaria Integrata, Verona, Italy); Giulia Bisoffi, Oscar Bortolami, Laura Crestani (Unità Supporto alla Ricerca e Biostatistica, Azienda Ospedaliera Universitaria Integrata, Verona, Italy); Fabiano Comperini (Italy); Ercole Concia, Emanuela Lattuada, Massimiliano Lanzafame, Paola Del Bravo (Infectious Diseases Section, Department of Pathology-Verona University Hospital - Veneto Region, Verona, Italy); Maddalena Cordioli, Fabio Rigo, Emanuele Guardalben, Ivan Marchesoni (Università degli studi di Verona, Verona, Italy); Barbara Suligoi, Vincenza Regine, Lucia Pugliese (Centro Operativo AIDS, Istituto Superiore di Sanità, Rome, Italy); Saulius Caplinskas, Irma Caplinskiene, Rima Krupenkaite (Centre for Communicable Diseases and AIDS, Vilnius, Lithuania); Gediminas Sargelis, Arturas Rudomanskis, ("Tolerant Youth Association", Vilnius, Lithuania); Sónia Dias, Ana Gama, Oriana Brás (Global Health and Tropical Medicine, GHTM, Instituto de Higiene e Medicina Tropical, IHMT, Universidade Nova de Lisboa, Portugal); João Piedade (Medical Microbiology Unit, Instituto de Higiene e Medicina Tropical, Lisbon, Portugal); Ricardo Fuertes, Nuno Pinto, João Brito, Júlio Esteves, Jesus Rojas, Fernando Ferreira, Miguel Rocha, Hugo Machado, Maria José Campos, (CheckpointLX, Portugal); Luís Mendão (GAT, Grupo Português de Ativistas sobre Tratamentos de VIH/SIDA - Pedro Santos, Portugal); Magdalena Rosińska, Bożena Kucharczyk, Marta NiedźwiedzkaStadnik, Łukasz Henszel, Andrzej Zieliński, Michał Czerwiński, (NIZP-PZH,

Warsaw, Poland); Michał Pawlęga, Ewelina Burdon, Małgorzata Gajdemska, Agnieszka Guściora, Nikodem Klasik, Katarzyna Rżanek, Michał Sawicki, Michał Tęcza, Ewelina Burdon, Małgorzata Gajdemska, Agnieszka Guściora, Nikodem Klasik, Katarzyna Rżanek, (Lambda Warszawa, Warsaw, Poland); Mateusz Dębski, Anna Maciejewska, Izabela Pazdan (SKA Warsaw, Poland); Izabela Pazdan (SKA, VCT side, Warsaw, Poland); Alexandru Rafila, Daniela Pitigoi, Adrian Abagiu (National Institute for infectious Diseases Prof. Dr. Matei Bals, Bucharest, Romania); Carolina Marin, loana Panzariu, Alexandru Miroiu, (ACCEPT Association, Bucharest, Romania); Madalina Popa, Monica Likker (National Institute for infectious Diseases Prof. Dr. Matei Bals, Bucharest, Romania); Maria Georgescu, Galina Musat, Dan Cojocaru, Mihai Lixandru, Raluca Teodorescu (Romanian Anti-AIDS Association - ARAS, Bucharest, Romania); Danica Staneková, Monika Hábeková, Tatiana Drobková, Zuzana Chabadová, Soňa Wimmerova, Maria Mojzesová (Slovak Medical University, NRC for HIV/AIDS prevention, Bratislava, Slovakia); Filip Kunč, Michal Skurák, Peter Bodnar, Katarína Horniaková, Mária Krahulcová, Jarmila Präsensová (Slovakia); Martin Smoleň, Peter Záhradník, Pavol Tibaj (NGO Dúhové srdce, Bratislava, Slovakia); Irena Klavs, Tanja Kustec, Claudia Adamič (National Institute of Public health, Ljubljana, Slovenia); Mario Poljak, Robert Krošelj, Jana Mlakar (Institute of Microbiology and Imunology, Medical faculty, University of Ljubljana, Ljubljana, Slovenia); Miran Šolinc (Association SKUC, Ljubljana,

Slovenia); Cinta Folch, Laia Ferrer, Alexandra Montoliu,Jordi Casabona, Anna Esteve, Montserrat Galdon (Centre for Epidemiological Studies on HIV/STI in Catalonia CEEISCAT, Agència de Salut Pública de Catalunya, Barcelona, Spain); Victoria Gonzalez (Microbiology Service, Hospital Universitari Germans Trias i 
Pujol, Barcelona, Spain); Rafael Muñoz (StopSida, Barcelona, Spain); Maria Axelsson, Torsten Berglund, Sharon Kuhlmann-Berenzon, Achilleas Tsoumanis, Inga Velicko, Christer Janson, Bartek Lindh, Kajsa Aperia (Public Health Agency of Sweden, Stockholm, Sweden); Buddha Babulanam, Hans Carlberg, Malte Davidsson, Nedo Entenza Gutierrez, Viktor Hildingsson, Henrik Klasson, Moises Peña Ramos, Cristian Quintero Rojas, Sven-Olof Sandberg, Andreas Samuelson Eric Sjöberg, Tommy Sjölund,Simon Svensson, Iván Valencia (Sweden); Filip Garcia, Olov Lindblad (RFSL Stockholm, Sweden); Jon Voss (Stockholm Gay Life, Sweden): Ronnie Ask, Anders Blaxhult, Maarit Maliniemi, Venhälsan, Stockholm South General Hospital, Stockholm, Sweden); Monica Ideström, Nils Blom (Public Health Agency of Sweden, Stockholm, Sweden); Nigel Sherriff, Christina Panton, Glynis Flood (School of Health Sciences, University of Brighton, Brighton, UK); Katrien Fransen, Tine Vermoesen (Aids reference laboratory, Institute of Tropical Medicine, Antwerp, Belgium); Ross Boseley, Marc Tweed (Terrence Higgins Trust, South, UK); Jonathon Roberts (Claude Nicol Centre, Royal Sussex County Hospital, Brighton, UK); Cinthia Menel Lemos (Executive Agency for Health and Consumers); Paolo Guglielmetti, Wolfgang Philipp, Matthias Schuppe (DG SANTE); Andrew Amato, Irina Dinca, Karin Haar, Anastasia Pharris, Teymur Noori (European Centre for Disease Prevention and Control ECDC); Igor Toskin, Armando Seuc, Natalie Maurer (Department of Reproductive Health \& Research of the World Health Organization WHO); Lev Zohrabyan, Alexandrina lovita, Maddalena Campioni, Patrick Noack (Joint United Nations Programme on HIV/AIDS UNAIDS); Rosanna Peeling (London School of Hygiene and Tropical Medicine); Lisa Johnston (USA).

\section{Availability of data and materials}

The self-administered questionnaire filled-in by the study participants is available as Additional file 1. The dataset used for the analysis presented in this manuscript is available as Additional file 2. The Stata syntax of the analysis is available as Additional file 3.

\section{Funding}

This document/paper is based on data from the Sialonll "Capacity building in combining targeted prevention with meaningful HIV surveillance amongst MSM" project, funded under the European Commission's (EC) Public Health Programme 2008-2013 (Work Plan 2010), reference number 100880. The sole responsibility lies with the authors of this document/paper and the Commission is not responsible for any use that may be made of the information contained therein. The funder had no role in the design of the study and collection, analysis, and interpretation of data and in writing the manuscript.

\section{Authors' contribution}

Most authors (UM, CN, MR, NS, LG, SFD, AFG, IA, EN, MM) participated in the design of the survey questionnaire and the organisation and implementation of the survey in the survey cities. IT was involved in the conception of the study and in the ethics approval by WHO. This analysis was conceived by UM. Data were analysed by UM, SBS. The first manuscript draft was written by UM. All authors (UM, CN, MR, NS, LG, SFD, AFG, IT, IA, EN, SBS, MM) contributed writing to the second and final draft. All authors approved the final draft.

\section{Ethics approval and consent to participate}

The study protocol was approved by the World Health Organization (WHO) Research Project Review Panel (RP2: A65- SIALON II: Capacity building in combining targeted prevention with meaningful HIV surveillance among men who have sex with men (MSM), Multi-country (Europe) protocol) and the WHO Research Ethics Review Committee (WHO-ERC: RPC557 - Capacity building in combining targeted prevention with meaningful HIV surveillance among MSM), and by ethics review committees in all participating countries (see list below). In all countries informed consent was obtained from the study participants. Ethics review committees in most countries required written informed consent from the participants, others such as Sweden and Germany required only verbal informed consent.

\begin{tabular}{lllll}
\hline Country & City & $\begin{array}{l}\text { Date of } \\
\text { approval }\end{array}$ & $\begin{array}{l}\text { Number of } \\
\text { the doc }\end{array}$ & $\begin{array}{l}\text { Name of the Ethics } \\
\text { Committee }\end{array}$ \\
\hline Belgium & Brussels & $\begin{array}{l}18 / 3 / \\
\text { ITG 860/13 }\end{array}$ & $\begin{array}{l}\text { Ethics Committee - } \\
\text { University of Antwerp }\end{array}$ \\
Bulgaria & Sofia & 2013 & & $\begin{array}{l}\text { Ethics Committee - } \\
\text { 26/3/ }\end{array}$ \\
& 2013 & & National Centre of
\end{tabular}

(Continued)

\begin{tabular}{|c|c|c|c|c|}
\hline Country & City & $\begin{array}{l}\text { Date of } \\
\text { approval }\end{array}$ & $\begin{array}{l}\text { Number of } \\
\text { the doc }\end{array}$ & $\begin{array}{l}\text { Name of the Ethics } \\
\text { Committee }\end{array}$ \\
\hline & & & & $\begin{array}{l}\text { Infectious and Parasitic } \\
\text { Diseases Sofia }\end{array}$ \\
\hline Germany & Hamburg & $\begin{array}{l}28 / 2 / \\
2013\end{array}$ & $\begin{array}{l}\mathrm{EA} 1 / 024 / \\
13\end{array}$ & $\begin{array}{l}\text { Ethics Committee - } \\
\text { Charité University }\end{array}$ \\
\hline Italy & Verona & $\begin{array}{l}22 / 05 / \\
2013\end{array}$ & $\begin{array}{l}\text { Prot.N.25334; } \\
\text { N.Prog. } 2341\end{array}$ & $\begin{array}{l}\text { Ethics Committee - } \\
\text { Verona University Hospital }\end{array}$ \\
\hline Lithuania & Vilnius & $\begin{array}{l}14 / 05 / \\
2013\end{array}$ & $\begin{array}{l}\text { N. } 158,200- \\
13-608-188\end{array}$ & $\begin{array}{l}\text { Regional Ethics Committee } \\
\text { Biomedical Research - } \\
\text { Vilnius }\end{array}$ \\
\hline Poland & Warsaw & $\begin{array}{l}18 / 4 / \\
2013\end{array}$ & $1 / 2013$ & Ethics Committee - Warsaw \\
\hline Portugal & Lisbon & $\begin{array}{l}14 / 6 / \\
2013\end{array}$ & 12-2013-PI & $\begin{array}{l}\text { Ethics Committee - Instituto } \\
\text { de Higiene e Medicina } \\
\text { Tropical }\end{array}$ \\
\hline Romania & Bucharest & $\begin{array}{l}18 / 04 / \\
2013\end{array}$ & C. 1937 & $\begin{array}{l}\text { Ethics Committee Institute } \\
\text { M. Bals - Bucharest }\end{array}$ \\
\hline Slovakia & Bratislava & $\begin{array}{l}16 / 05 / \\
2013\end{array}$ & - & $\begin{array}{l}\text { Ethics Committee of the } \\
\text { Slovak Medical University }\end{array}$ \\
\hline Slovenia & Ljubljana & $\begin{array}{l}16 / 4 / \\
2013\end{array}$ & $87 / 04 / 13$ & $\begin{array}{l}\text { Ethics Committee Republic } \\
\text { of Slovenia }\end{array}$ \\
\hline Spain & Barcelona & $\begin{array}{l}17 / 4 / \\
2013\end{array}$ & PI 13014 & $\begin{array}{l}\text { Ethics Committee - } \\
\text { Germans Trias i Pujol } \\
\text { Hospital }\end{array}$ \\
\hline Sweden & Stockholm & $\begin{array}{l}14 / 5 / \\
2013\end{array}$ & $\begin{array}{l}\text { 2013/3:5- } \\
2013-05-02\end{array}$ & $\begin{array}{l}\text { Ethics Committee - } \\
\text { Folkhalsomyndigheten }\end{array}$ \\
\hline UK & Brighton & $\begin{array}{l}26 / 01 / \\
2013\end{array}$ & $\begin{array}{l}\text { FREGC-13- } \\
001 . R 1\end{array}$ & $\begin{array}{l}\text { Faculty of Health and Social } \\
\text { Science Research Ethics and } \\
\text { Governance Committee }\end{array}$ \\
\hline
\end{tabular}

Consent for publication

Not applicable.

\section{Competing interests}

The authors declare that they have no competing interests.

\section{Publisher's Note}

Springer Nature remains neutral with regard to jurisdictional claims in published maps and institutional affiliations.

\section{Author details}

'Department of Infectious Diseases Epidemiology, Robert Koch-Institute, Berlin, Germany. ${ }^{2}$ Department of Public Health, Institute of Tropical Medicine, Antwerp, Belgium. ${ }^{3}$ Department of Epidemiology, National Institute of Public Health, Warsaw, Poland. ${ }^{4}$ Health Sciences, University of Brighton, Brighton, UK. ${ }^{5}$ Infectious Diseases Section, Department of Diagnostics and Public Health, University of Verona, Verona, Italy. ${ }^{6}$ Escola Nacional de Saúde Pública Universidade, Centro de Investigação em Saúde Pública, Universidade Nova de Lisboa, Lisbon, Portugal. ${ }^{7}$ Instituto de Higiene e Medicina Tropical, Global Health and Tropical Medicine, Universidade Nova de Lisboa, Lisbon, Portugal. ${ }^{8}$ Department of Reproductive Health and Research, World Health Organization, Geneva, Switzerland. ${ }^{9}$ National Centre of Infectious and Parasitic Diseases, National Reference Laboratory of HIV, Sofia, Bulgaria. ${ }^{10}$ Ministry of Health, Program "Prevention and control of HIV/AIDS", Sofia, Bulgaria. 


\section{Received: 14 February 2018 Accepted: 10 July 2018} Published online: 06 August 2018

\section{References}

1. WHO Europe. Action plan for the health sector response to HIV in the WHO European region. Copenhagen: WHO Europe; 2016. URL: http://www.euro. who.int/en/publications/abstracts/action-plan-for-the-health-sectorresponse-to-hiv-in-the-who-european-region-2017

2. Brown AE, Attawell K, Hales D, Rice B, Pharris A, Noori T. Monitoring the HIV continuum of care in key populations across Europe and Central Asia. HIV Med. 2018; https://doi.org/10.1111/hiv.12603. [Epub ahead of print].

3. UNAIDS: 90-90-90. An ambitious treatment target to help end the aids epidemic. UNAIDS; 2014. URL: http://www.unaids.org/en/resources/ documents/2017/90-90-90

4. Kall MM, Smith RD, Delpech VC. Late HIV diagnosis in Europe: a call for increased testing and awareness among general practitioners. Eur. J. Gen. Pract. 2012;18(3):181-6.

5. Schwarcz S, Richards TA, Frank H, Wenzel C, Hsu LC, Chin CS, Murphy J, Dilley J. Identifying barriers to HIV testing: personal and contextual factors associated with late HIV testing. AIDS Care. 2011;23(7):892-900.

6. Supervie V, Ndawinz JD, Lodi S, Costagliola D. The undiagnosed HIV epidemic in France and its implications for HIV screening strategies. AIDS. 2014;28(12):1797-804.

7. Medland NA, Nicholson S, Chow EPF, Read TRH, Bradshaw CS, Denham I, Fairley CK. Time from HIV infection to virological suppression: dramatic fall from 2007 to 2016. AIDS. 2017:31(17):2377-85.

8. Deblonde J, De Koker P, Hamers FF, Fontaine J, Luchters S, Temmerman M. Barriers to HIV testing in Europe: a systematic review. Eur J Pub Health. 2010;20(4):422-32

9. Mocroft A, Lundgren JD, Sabin ML, Monforte A, Brockmeyer N, Casabona J, Castagna A, Costagliola D, Dabis F, De Wit S, et al. Risk factors and outcomes for late presentation for HIV-positive persons in Europe: results from the collaboration of observational HIV epidemiological research Europe study (COHERE). PLoS Med. 2013;10(9):e1001510.

10. Meireles P, Lucas R, Carvalho C, Fuertes R, Brito J, Campos MJ, Mendao L, Barros $\mathrm{H}$. Incident risk factors as predictors of HIV seroconversion in the Lisbon cohort of men who have sex with men: first results, 2011-2014. Euro Surveil. 2015;20(14)

11. Marcus U, Ort J, Grenz M, Eckstein K, Wirtz K, Wille A. Risk factors for HIV and STI diagnosis in a community-based HIV/STI testing and counselling site for men having sex with men (MSM) in a large German city in 2011-2012. BMC Infect Dis. 2015;15:14.

12. Santos-Hövener C, Zimmermann R, Kucherer C, Bätzing-Feigenbaum J, Wildner S, Hamouda O, Marcus U. Conversation about Serostatus decreases risk of acquiring HIV: results from a case control study comparing MSM with recent HIV infection and HIV negative controls. BMC Public Health. 2014;14:453.

13. Diez M, Bleda MJ, Varela JR, Ordonana J, Azpiri MA, Vall M, Santos C, Viloria $L$, de Armas $C$, Urena JM, et al. Trends in HIV testing, prevalence among first-time testers, and incidence in most-at-risk populations in Spain: the EPIVIH study, 2000 to 2009. Euro Surveil. 2014;19(47):20971.

14. Giuliani M, Vescio MF, Latini A, Palamara G, Pimpinelli F, Dona MG, Stivali F, Carduccelli F, Ensoli F, Di Carlo A, et al. Continuous increase in HIV-1 incidence after the year 2000 among men who have sex with men in Rome: insights from a 25-year retrospective cohort study. Eurosurveillance. 2014;19(47):20969.

15. Ferrer L, Furegato M, Foschia JP, Folch C, Gonzalez V, Ramarli D, Casabona J, Mirandola M. Undiagnosed HIV infection in a population of MSM from six European cities: results from the sialon project. Eur J Pub Health. 2015;25(3): 494-500.

16. Saxton PJ, Dickson NP, Griffiths R, Hughes AJ, Rowden J: Actual and undiagnosed HIV prevalence in a community sample of men who have sex with men in Auckland, New Zealand BMC Public Health 2012, 12(1):92.

17. Gama A, Abecasis A, Pingarilho M, Mendao L, Martins MO, Barros H, Dias S Cruising venues as a context for HIV risky behavior among men who have sex with men. Arch Sex Behav. 2017:46(4):1061-8.

18. Baral S, Logie CH, Grosso A, Wirtz AL, Beyrer C. Modified social ecological model: a tool to guide the assessment of the risks and risk contexts of HIV epidemics. BMC Public Health. 2013;13(1):482.

19. de Wit JB, Adam PC. To test or not to test: psychosocial barriers to HIV testing in high-income countries. HIV Med. 2008;9(Suppl 2):20-2.
20. Adam PC, de Wit JB, Bourne CP, Knox D, Purchas J. Promoting regular testing: an examination of HIV and STI testing routines and associated socio-demographic, behavioral and social-cognitive factors among men who have sex with men in New South Wales, Australia. AIDS Behav. 2014; 18(5):921-32.

21. Gios L, Mirandola M, Toskin I, Marcus U, Dudareva-Vizule S, Sherriff N, Breveglieri M, Furegato M, Folch C, Ferrer L, et al. Bio-behavioural HIV and STI surveillance among men who have sex with men in Europe: the sialon II protocols. BMC Public Health. 2016;16:212.

22. Rabe-Hesketh S, Skrondal A: Multilevel and longitudinal modeling using stata, 2nd edn. College Station, Tex.: Stata Press Publication; 2008. URL: Publisher description http://www.loc.gov/catdir/enhancements/fy0917/ 2009419524-d.html

23. Mirandola M, Gios L, Sherriff N, Toskin I, Marcus U, Schink SB, Suligoi B, Folch C, Rosińska M (eds.): the sialon II project. Report on a bio-behavioural survey among MSM in 13 European cities Cierre Grafica; 2016. URL: http:// www.sialon.eu/data2/file/133_Sialon\%20ll_Report\%20on\%20a\%20Biobehavioural\%20Survey\%20amo ng\%20MSM\%20in\%2013\%20European\%20cities.pdf

24. Dudareva-Vizule S, Marcus U. Sialon II formative research report. Berlin: Robert Koch-Institut; 2013. URL: http://www.sialon.eu/data2/file/133_347_ SIALON_FR_report_final.pdf

25. van Sighem A, Vidondo B, Glass TR, Bucher HC, Vernazza P, Gebhardt M, de Wolf F, Derendinger S, Jeannin A, Bezemer D, et al. Resurgence of HIV infection among men who have sex with men in Switzerland: mathematical modelling study. PLoS One. 2012;7(9):e44819.

26. Mammone A, Pezzotti P, Regine V, Camoni L, Puro V, Ippolito G, Suligoi B, Girardi E. How many people are living with undiagnosed HIV infection? An estimate for Italy, based on surveillance data. AIDS. 2016;30(7):1131-6.

27. Rosinska M, Gwiazda P, De Angelis D, Presanis AM. Bayesian evidence synthesis to estimate HIV prevalence in men who have sex with men in Poland at the end of 2009. Epidemiol Infect. 2015;144(6):1175-91.

28. Dijkstra M, de Bree GJ, Stolte IG, Davidovich U, Sanders EJ, Prins M, Schim van der Loeff MF. Development and validation of a risk score to assist screening for acute HIV-1 infection among men who have sex with men. BMC Infect Dis. 2017;17:425.

29. Lin TC, Gianella S, Tenenbaum T, Little SJ, Hoenigl M, Simple Symptom A. Score for acute HIV infection in a San Diego community based screening program. Clin Infect Dis. 2017;67(1):105-11.

30. Rosinska M, Gios L, Nostlinger C, Vanden Berghe W, Marcus U, Schink S, Sherriff N, Jones AM, Folch C, Dias S, et al. Prevalence of drug use during sex amongst MSM in Europe: results from a multi-site bio-behavioural survey. Int J Drug Policy. 2018;55:231-41.

31. Bellan SE, Dushoff J, Galvani AP, Meyers LA. Reassessment of HIV-1 acute phase infectivity: accounting for heterogeneity and study design with simulated cohorts. PLoS Med. 2015;12(3):e1001801

32. Rutstein SE, Ananworanich J, Fidler S, Johnson C, Sanders EJ, Sued O, SaezCirion A, Pilcher CD, Fraser C, Cohen MS, et al. Clinical and public health implications of acute and early HIV detection and treatment: a scoping review. J Int AIDS Soc. 2017;20(1):21579.

33. Abu-Raddad LJ. Role of acute HIV infection in driving HIV transmission: implications for HIV treatment as prevention. PLoS Med. 2015;12(3): e1001803.

34. Feinstein BA, Johnson BA, Parsons JT, Mustanski B. Reactions to testing HIV negative: measurement and associations with sexual risk behaviour among young MSM who recently tested HIV negative. AIDS Behav. 2017;21(5):1467-77.

35. Hoenigl M, Anderson CM, Green N, Mehta SR, Smith DM, Little SJ. Repeat HIV-testing is associated with an increase in behavioral risk among men who have sex with men: a cohort study. BMC Med. 2015:13:218.

36. Marcus U, Gassowski M, Kruspe M, Drewes J. Recency and frequency of HIV testing among men who have sex with men in Germany and sociodemographic factors associated with testing behaviour. BMC Public Health. 2015;15:727

37. Pant Pai N, Sharma J, Shivkumar S, Pillay S, Vadnais C, Joseph L, Dheda K, Peeling RW. Supervised and unsupervised self-testing for HIV in high- and low-risk populations: a systematic review. PLoS Med. 2013;10(4):e1001414.

38. Champenois K, Le Gall J-M, Jacquemin C, Jean S, Martin C, Rios L, Benoit O, Vermoesen S, Lert F, Spire B, et al. ANRS-COM'TEST: description of a community-based HIV testing intervention in non-medical settings for men who have sex with men. BMJ Open. 2012;2(2):e000693. 
39. Thornton AC, Delpech V, Kall MM, Nardone A. HIV testing in community settings in resource-rich countries: a systematic review of the evidence. HIV Med. 2012;13(7):416-26.

40. McCormack S, Dunn DT, Desai M, Dolling DI, Gafos M, Gilson R, Sullivan AK, Clarke A, Reeves I, Schembri G, et al. Pre-exposure prophylaxis to prevent the acquisition of HIV-1 infection (PROUD): effectiveness results from the pilot phase of a pragmatic open-label randomised trial. Lancet. 2016; 387(10013):53-60.

41. Grant RM, Lama JR, Anderson PL, McMahan V, Liu AY, Vargas L, Goicochea P, Casapia M, Guanira-Carranza JV, Ramirez-Cardich ME, et al. Preexposure chemoprophylaxis for HIV prevention in men who have sex with men. N Engl J Med. 2010;363(27):2587-99.

42. Molina JM, Capitant C, Spire B, Pialoux G, Cotte L, Charreau I, Tremblay C, Le Gall JM, Cua E, Pasquet A, et al. On-demand Preexposure prophylaxis in men at high risk for HIV-1 infection. N Engl J Med. 2015;373(23):2237-46.

Ready to submit your research? Choose BMC and benefit from:

- fast, convenient online submission

- thorough peer review by experienced researchers in your field

- rapid publication on acceptance

- support for research data, including large and complex data types

- gold Open Access which fosters wider collaboration and increased citations

- maximum visibility for your research: over $100 \mathrm{M}$ website views per year

At $\mathrm{BMC}$, research is always in progress.

Learn more biomedcentral.com/submissions 